This item was submitted to Loughborough's Research Repository by the author.

Items in Figshare are protected by copyright, with all rights reserved, unless otherwise indicated.

\title{
Influence of anti-dive and anti-squat geometry in combined vehicle bounce and pitch dynamics
}

PLEASE CITE THE PUBLISHED VERSION

PUBLISHER

Professional Engineering Publishing / @ IMECHE

VERSION

VoR (Version of Record)

LICENCE

CC BY-NC-ND 4.0

\section{REPOSITORY RECORD}

Azman, M., Homer Rahnejat, P.D. King, and T.J. Gordon. 2019. "Influence of Anti-dive and Anti-squat Geometry in Combined Vehicle Bounce and Pitch Dynamics". figshare. https://hdl.handle.net/2134/4829. 
This item was submitted to Loughborough's Institutional Repository (https://dspace.lboro.ac.uk/) by the author and is made available under the following Creative Commons Licence conditions.

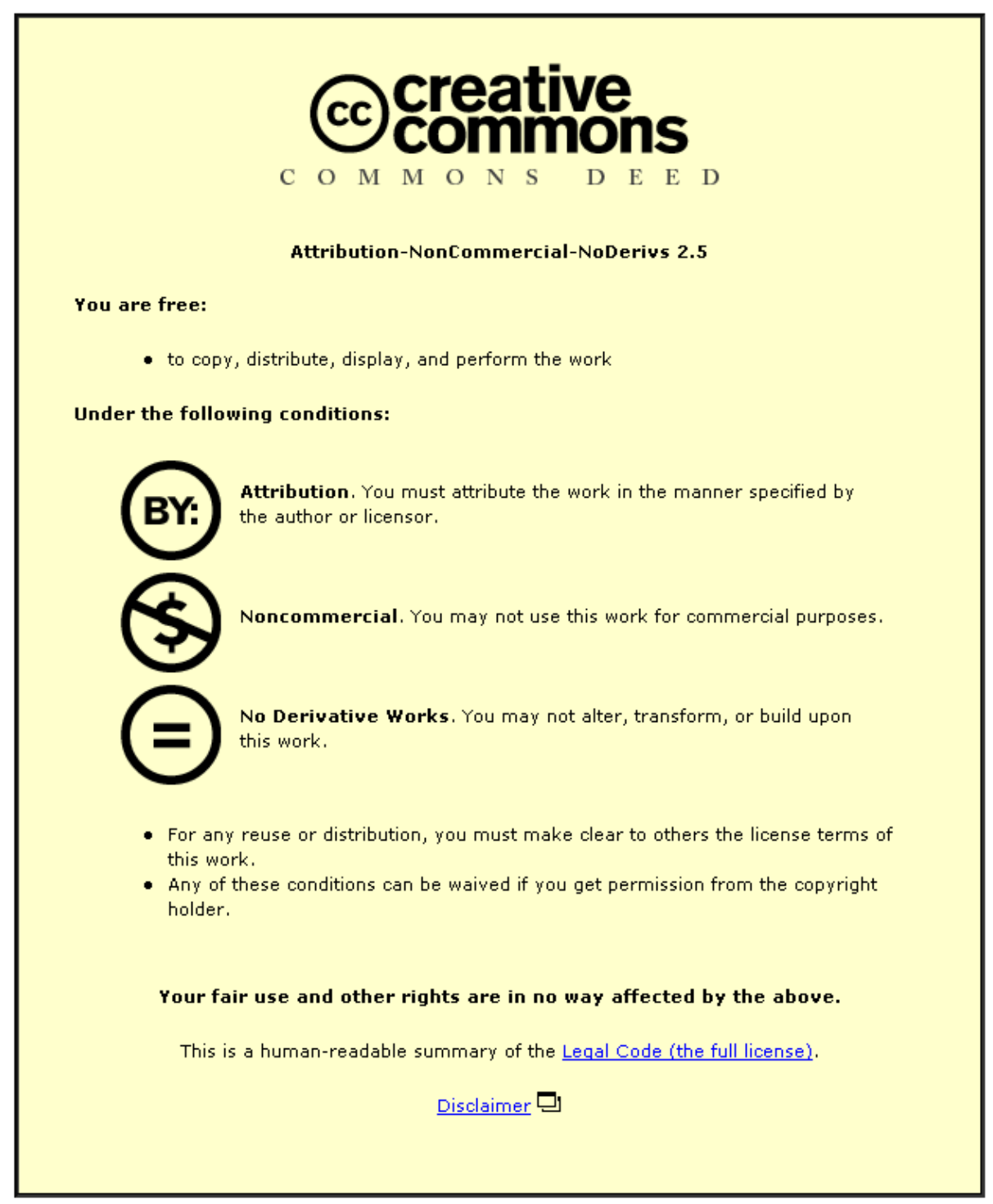

For the full text of this licence, please go to: http://creativecommons.org/licenses/by-nc-nd/2.5/ 


\title{
Influence of anti-dive and anti-squat geometry in combined vehicle bounce and pitch dynamics
}

\author{
M Azman ${ }^{1}$, H Rahnejat ${ }^{1 *}$, P D King ${ }^{1}$ and T J Gordon ${ }^{2}$ \\ ${ }^{1}$ Wolfson School of Mechanical and Manufacturing Engineering, Loughborough University, Loughborough, UK \\ ${ }^{2}$ Transportation Research Institute, University of Michigan, Ann Arbor, Michigan, USA
}

\begin{abstract}
The paper presents a six-degree-of-freedom (6-DOF) multi-body vehicle model, including realistic representation of suspension kinematics. The suspension system comprises anti-squat and antidive element. The vehicle model is employed to study the effect of these features upon combined bounce and pitch plane dynamics of the vehicle, when subjected to bump riding events. The investigations are concerned with a real vehicle and the numerical predictions show reasonable agreement with measurements obtained on an instrumented vehicle under the same manoeurves.
\end{abstract}

Keywords: vehicle dynamics, principle of virtual work, multi-body dynamics, anti-squat and anti-dive characteristics

NOTATION
$\boldsymbol{A}_{1 \ldots 4}$
$\boldsymbol{B}_{1,2,3}$
$\boldsymbol{e}_{2}$
$\boldsymbol{e}_{3}$
$\boldsymbol{F}_{\mathrm{a}}$
$\boldsymbol{F}_{x 1}, \ldots, \boldsymbol{F}_{x 4}$
$\boldsymbol{F}_{y 1}, \ldots, \boldsymbol{F}_{y 4}$
$\boldsymbol{F}_{z 1}, \ldots, \boldsymbol{F}_{z 4}$
$\boldsymbol{F}_{\mathrm{t}}^{\text {max }}$
$\boldsymbol{F}_{\text {aero }}$
$\boldsymbol{F}_{\text {tyres }}$
$\boldsymbol{F}_{\text {weight }}$
$g$
$G$
$\boldsymbol{I}_{x x, y y, z z}$
$\boldsymbol{I}_{x z}$
$\boldsymbol{I}_{G}$

The MS was received on 2 February 2004 and was accepted after revision for publication on 6 September 2004.

* Corresponding author: Wolfson School of Mechanical and Manufacturing Engineering, University of Loughborough, Loughborough, Leicestershire LE11 3TU, UK.
$I_{3}$

$k_{\text {aero }}$

$k$ of $\boldsymbol{M}_{1}$ respectively

matrices $\boldsymbol{M}_{6}, \boldsymbol{M}_{7}$ and $\boldsymbol{M}_{8}$ multiplied by the inverse of $\boldsymbol{M}_{1}$

$y$ axis base vector for the tyre coordinate system unit vector (upward) normal to the road surface at $S$

actual tyre forces

longitudinal tyre forces

lateral tyre forces

vertical tyre forces

nominal maximum 'rim contact' tyre force aerodynamic force

tyre forces

vehicle weight

gravity

vehicle centre of gravity

roll, pitch and yaw moment of inertia

about the mass centre

product of inertia

inertia matrix of vehicle
KI, KP

M

$\mathbf{M}_{\mathrm{tyres}}$

$\mathbf{M}_{1}$

$\mathbf{M}_{2}, \mathbf{M}_{3}, \mathbf{M}_{4}$

$\mathbf{M}_{5}$

$\mathbf{M}_{6}$

$\mathbf{M}_{7}$

$\mathbf{M}_{8}$

$n_{\text {B }}$

$n_{S}$

$p, q, r$

P

$Q$

$\boldsymbol{r}_{\mathrm{A}}\left(z_{\text {sus }}\right)$

$\boldsymbol{r}_{G}$ $n \times n$ identity matrix

aerodynamic drag coefficient unit vector of the global $z$ direction, relative to the vehicle coordinates integral and proportional gains vehicle mass moment about $G$ from the tyre forces generalized mass matrix matrix coefficients arising from the bilinear gyroscopic terms matrix coefficient from aerodynamic drag matrix consisting of the sum of all the applied forces and body dimensions and giving the main contributions from the tyre force inputs

matrix containing the moment effect of dynamic suspension deflections $\tilde{z}$ matrix containing the gravity term unit vector in body $z$ unit vector (upward) normal to the road surface at $S$ angular velocity in the $x, y$ and $z$ axis respectively nominal contact patch centre new position of $P$ obtained by translating $Q$ in the body $z$ axes

kinematics term accounting for the steering torque distance of the contact patches from the centre of gravity 
$r_{p}$

$\boldsymbol{r}_{p}^{\{\mathrm{B}\}}$

$r_{S}$

R

$\mathbf{R}_{1,2,3}$

$\mathbf{R}^{\{\mathrm{B} \rightarrow \mathrm{G}\}}$

$S, S_{\text {ref }}$

$T_{\mathrm{d}}$

$U, V$

$v$

$v_{G}$

$v_{Q}$

$x, \dot{x}$

$x_{P}, y_{P}$

$x_{4}$

$y_{1}$

$y_{2}$

$z_{\mathrm{s}}$

$Z_{\mathrm{t}}$

$z_{\mathrm{t}}^{\max }$

$z_{\mathrm{t}}^{\min }$

$z_{4}$

$\tilde{z}$

$\beta$

$\gamma$

$\delta x, \delta y$

$\delta z$

$\delta v$

$\delta$

$\delta_{\mathrm{k}}\left(z_{\mathrm{sus}}\right)$

$\varepsilon_{1}$

$\varepsilon_{2}$

$\theta_{\mathrm{v}}$

$\theta_{1}, \theta_{2}, \theta_{3}$

$\dot{\theta}_{1}, \dot{\theta}_{2}, \dot{\theta}_{3}$ position of the nominal contact patch centre in the global coordinates

position of the nominal contact patch

centre in the vehicle body coordinates

based at $G$

position of the nominal contact patch on

the road surface

actual vehicle orientation

orientation matrix in the roll, pitch and

yaw axes

passive rotation matrix that converts from

body to global coordinates, using Euler

angles

actual and desired speed

drive torque (assumed to be generated

from an inboard differential)

longitudinal and lateral velocity

unit vector normal to the wheel plane

three components of translational

velocity

velocity of point $Q$ moving within the

plane (road surface)

state and state derivative variables

position of point $P$ from the centre of

gravity in the $x-y$ plane

set of $x$ coordinates at the four tyre

contact patches

acceleration/brake command

steering angle

suspension deflection

tyre deflection

loss of tyre contact, where $F_{\mathrm{t}} \rightarrow 0$

maximum tyre compression

set of $z$ coordinates at the four tyre

contact patches from the centre of gravity

suspension deflections

vehicle direction (yaw angle)

lateral inclination angle

contact patch forward progression and

lateral scrub respectively

suspension vertical changes

change in the caster angle

static toe angle

kinematics term accounting for bump-steer

deviation of actual speed from desired

speed

directional error between where the

car is pointing and where it should be

going

angle of the reference vector of the

vehicle in global coordinates

roll angle, pitch angle and yaw angle

respectively

derivative of roll angle, pitch angle and

yaw angle respectively $\lambda$

$\mu$

$v \rho$

$\rho\left(z_{\text {sus }}\right)$

$\phi$

$\varphi, \theta, \psi$

$\omega_{1}, \omega_{2}, \omega_{3}$

$\omega$

sum of the suspension and tyre

deflections

expansion velocity of the suspension-

tyre combination

caster and camber angle

kinematics term accounting for bump-

camber

actual steer angle

roll angle, pitch angle and yaw angle

respectively

body angular velocity, roll, pitch and yaw

axis respectively

three components of angular velocity

\section{INTRODUCTION}

During the last decade, improvements in computer capabilities and commercial multi-body simulation software have led to a tendency to develop detailed modelling of vehicle systems. Such software is based on physical representations, usually requiring large quantities of input data $[\mathbf{1 , 2}]$. These are not always readily available to all engineering analysts. Even when the full set of input parameters is available, the simulation studies run considerably slower than the customized programs, which are less complex but adequate for the purpose of investigation. The complexity of large models can sometimes reduce the reliability of simulation, especially when the model is constructed during the hectic process of development and design [2]. Such circumstances often result in simulation projects that can only confirm the design and measurement but seldom contribute to a better design before various test vehicles are built. As reported in references [2] and [3], various problems concerning the dynamics of a vehicle can be reliably solved with comparatively simple models of the real system. However, simple models have their limits and are only suitable for certain types of test. The work carried out in this paper is the initial work to establish the limits of validity of a functional vehicle model that is capable of evaluating handling analysis as well as ride comfort, such as bump riding events. These simpler multi-body models are regarded as intermediate [4].

The model reported here is used to investigate the effect of anti-squat and anti-dive geometry in response to road profile inputs. As Sharp [5] has already pointed out, transient dynamics of vehicles is a non-trivial problem, even for a standard road car, and a simple manoeuvre such as accelerating or braking on a flat road, the so-called standard analysis, therefore, is severely limited in its applicability. This paper consisders a real-world scenario including both transient torque inputs and vertical road surface geometry. One question addressed is the adequacy of a simplified system model in predicting these effects. A second question is the effectiveness of anti-squat and anti-dive geometry on the pitch plane dynamics under such complex real-world conditions. The model reported here has a 
six-degree-of-freedom (6-DOF) vehicle body with realistic suspension kinematics and a non-linear load-dependent tyre model. The results of the analysis for a given test are compared with the measured performance data from the actual vehicle.

\section{DESCRIPTION OF AN INTERMEDIATE VEHICLE MODEL}

The model can be divided into five main modules: rigid body dynamics, vehicle kinematics, suspension and steering, driveline and tyres and a driver model.

\subsection{Rigid body dynamics}

This module uses a body-centred coordinate system. The inputs are the 12 tyre force components

$$
F_{\text {tyres }}=\left[F_{x 1}, \ldots, F_{x 4}, F_{y 1}, \ldots, F_{y 4}, F_{z 1}, \ldots, F_{z 4}\right]^{\mathrm{T}}
$$

These are applied directly to the vehicle body. This is justified because the unsprung mass is neglected and the resultant forces and moments on the unsprung mass equilibrate. Therefore, the forces and moments are directly 'transmitted' to the vehicle body structure. The state variables are the mass centre translational and rigid body angular velocities using the body-fixed SAE (Society of Automotive Engineers) frame of reference

$$
\boldsymbol{x}=[U, V, W, p, q, r]^{\mathrm{T}}
$$

Other inputs include the aerodynamic force (applied at the centre of gravity of the sprung mass)

$$
\boldsymbol{F}_{\text {aero }}=-k_{\text {aero }} \boldsymbol{v}_{G} \boldsymbol{v}_{G}
$$

(where $\boldsymbol{v}_{G}=[U, V, W]^{\mathrm{T}}$ ) and the vehicle weight $\boldsymbol{F}_{\text {weight }}=$ $M g k$, where $\boldsymbol{k}$ is the unit vector of the global $z$ direction

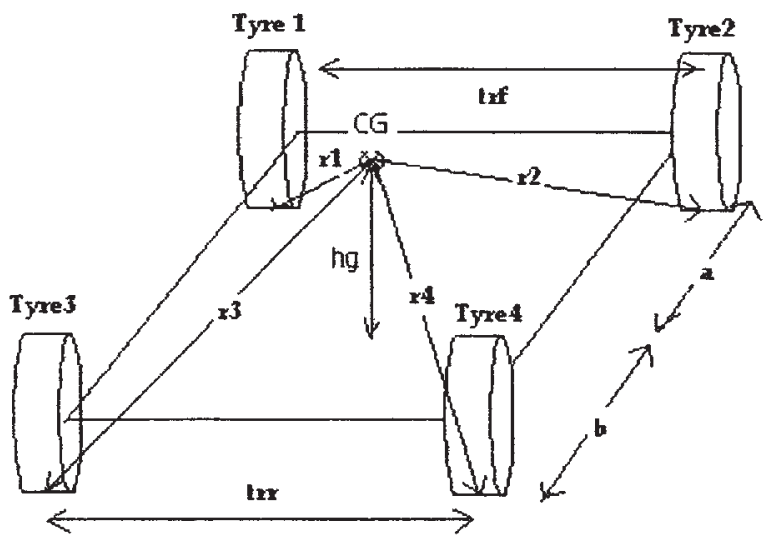

Fig. 1 A schematic representation of the intermediate vehicle model relative to the vehicle coordinates. Equations of motion are based on the standard Newton-Euler form

$$
\begin{aligned}
& M\left(\dot{\boldsymbol{v}}_{G}^{\text {rel }}+\omega \times \boldsymbol{v}_{\mathrm{G}}\right)=\boldsymbol{F}_{\text {aero }}+\Sigma \boldsymbol{F}_{\text {tyres }}+\boldsymbol{F}_{\text {weight }} \\
& \mathbf{I}_{G} \boldsymbol{\omega}^{\text {rel }}+\omega \times\left(\boldsymbol{I}_{\mathrm{G}} \omega\right)=\Sigma \mathbf{M}_{\text {tyres }}
\end{aligned}
$$

Therefore, the intermediate model has six degrees of freedom. They include vehicle translation along the $x$ and $y$ directions, bounce the $z$ direction and roll, pitch and yaw about these axes respectively. The analysis carried out in this paper is for straight-line motions involving the degrees of freedom $x, z$ and pitch motion. The model is, however, generic and can be used for other manoeuvres such as combined cornering and braking, and single-event bump riding; involving appreciable vehicle roll.

The inertial matrix assumes lateral symmetry in the vehicle model

$$
\mathbf{I}_{G}=\left(\begin{array}{ccc}
I_{x x} & 0 & -1_{x z} \\
0 & I_{y y} & 0 \\
-I_{x z} & 0 & I_{z z}
\end{array}\right)
$$

The equations of motion can be rewritten in terms of the state variables in the following form

$$
\begin{aligned}
\mathbf{M}_{1} \dot{\boldsymbol{x}}= & \left(p \mathbf{M}_{2}+q \mathbf{M}_{3}+r \mathbf{M}_{4}+\mathbf{M}_{5}\left|\mathbf{v}_{G}\right|\right) \boldsymbol{x}+\mathbf{M}_{6} \boldsymbol{F}_{\text {tyres }} \\
& +\mathbf{M}_{7} \tilde{\boldsymbol{z}} \otimes \boldsymbol{F}_{x y}+\mathbf{M}_{8} \boldsymbol{F}_{\text {weight }}
\end{aligned}
$$

where, for example

$$
\mathbf{M}_{1}=\left(\begin{array}{cc}
M \mathbf{I}_{3} & \mathbf{0}_{3 \times 3} \\
\mathbf{0}_{3 \times 3} & \mathbf{I}_{G}
\end{array}\right)
$$

and $\mathbf{M}_{\mathbf{1}}$ is a generalized mass matrix. Here, $\mathbf{0}_{n \times m}$ is an $n \times m$ matrix of zeros, $\mathbf{I}_{n \times m}$ similarly denotes a matrix of unity values and $\mathbf{I}_{\mathbf{3}}$ is an $n \times n$ identity matrix; $\mathbf{M}_{\mathbf{2}}, \mathbf{M}_{\mathbf{3}}$ and $\mathbf{M}_{\mathbf{4}}$ contain coefficients arising from the bilinear gyroscopic terms (those obtained from products of the form $\omega \times \cdots$ terms in the above equations of motion), with $\mathbf{M}_{\mathbf{2}}$ picking up all the terms in $p, \mathbf{M}_{\mathbf{3}}$ picking up all the remaining terms in $q$ and $\mathbf{M}_{\mathbf{4}}$ providing the remaining $r$ terms (see the Notation for full details); $\mathbf{M}_{\mathbf{5}}$ relates to the aerodynamic drag (and has zeros in rows 4 to 6, since no aerodynamic moments are included) and $\mathbf{M}_{\mathbf{6}}$ consists of ones (to sum all the applied forces) and body dimensions (to evaluate moments) and gives the main contributions from the tyre force inputs

$$
\mathbf{M}_{6}=\left(\begin{array}{ccc}
\mathbf{1}_{4 \times 1} & \mathbf{0}_{4 \times 1} & \mathbf{0}_{4 \times 1} \\
\mathbf{0}_{4 \times 1} & \mathbf{1}_{4 \times 1} & \mathbf{1}_{4 \times 1} \\
\mathbf{0}_{4 \times 1} & \mathbf{0}_{4 \times 1} & \mathbf{1}_{4 \times 1} \\
\mathbf{0}_{4 \times 1} & -z_{4} & \boldsymbol{y}_{4} \\
\boldsymbol{z}_{4} & \mathbf{0}_{4 \times 1} & -\boldsymbol{x}_{4} \\
-\boldsymbol{y}_{4} & \boldsymbol{x}_{4} & \mathbf{0}_{4 \times 1}
\end{array}\right)
$$

where $\boldsymbol{x}_{4}=[a a-b-b]$ is the set of $x$ coordinates at the four tyre contact patches (see Fig. 1). The $z$ coordinates are all equal to the mass centre height of the vehicle in its 
trim condition, $z_{4}=\left[h_{G} h_{G} h_{G} h_{G}\right]$, except for the moment effect of dynamic suspension deflections $\tilde{z}$, which are picked up by the $\mathbf{M}_{\mathbf{7}}$ terms

$$
\mathbf{M}_{7}=\left(\begin{array}{cc}
\mathbf{0}_{3 \times 4} & \mathbf{0}_{3 \times 4} \\
\mathbf{0}_{1 \times 4} & -\mathbf{1}_{1 \times 4} \\
\mathbf{1}_{1 \times 4} & \mathbf{0}_{1 \times 4} \\
\mathbf{0}_{1 \times 4} & \mathbf{0}_{1 \times 4}
\end{array}\right)
$$

Also note that

$$
\begin{gathered}
\tilde{\boldsymbol{z}} \otimes \boldsymbol{F}_{x y} \equiv\left[\tilde{z}_{1} F_{x 1}, \tilde{z}_{2} F_{x 2}, \tilde{z}_{3} F_{x 3}, \tilde{z}_{4} F_{x 4}, \tilde{z}_{1} F_{y 1}, \tilde{z}_{2} F_{y 2},\right. \\
\left.\tilde{z}_{3} F_{y 3}, \tilde{z}_{4} F_{y 4}\right]^{\mathrm{T}}
\end{gathered}
$$

Finally, the gravity term is included by

$$
\mathbf{M}_{8}=\left(\begin{array}{c}
\mathbf{I}_{3} \\
\mathbf{0}_{3 \times 3}
\end{array}\right)
$$

To evaluate state variable derivatives, the matrices on the right-hand side of equation (4) are multiplied by $\mathbf{M}_{1}^{-1}$ to give the form

$$
\begin{aligned}
\dot{\boldsymbol{x}}= & \left(p \mathbf{A}_{1}+q \mathbf{A}_{2}+r \mathbf{A}_{3}+\mathbf{A}_{4}\left|\mathbf{v}_{G}\right|\right) \boldsymbol{x}+\mathbf{B}_{1} \boldsymbol{F}_{\mathrm{T}} \\
& +\mathbf{B}_{2} \tilde{\boldsymbol{z}} \otimes \boldsymbol{F}_{x y}+\mathbf{B}_{3} \boldsymbol{F}_{\text {weight }}
\end{aligned}
$$

where $\mathbf{A}_{1}=\mathbf{M}_{1}^{-1} \mathbf{M}_{2}$, etc.

\subsection{Vehicle kinematics}

The main purpose is to turn the local (i.e. the vehicle-based) angular velocities into Euler angle derivatives and then integrate these to find roll, pitch and yaw angles. Following the equations given in references [6] and [7], the Euler angles are $\theta_{1}=\varphi, \theta_{2}=\theta$ and $\theta_{3}=\psi$ (roll, pitch and yaw respectively) and applied in the sequential order yaw, pitch and roll in a body-fixed frame of reference to give the (active) transformation matrix from reference to actual vehicle orientation as

$$
\mathbf{R}=\mathbf{R}_{3}\left(\theta_{3}\right) \mathbf{R}_{2}\left(\theta_{2}\right) \mathbf{R}_{1}\left(\theta_{1}\right)
$$

Note that the order is reversed here since each matrix is relative to the local body axes. Thus

$$
\begin{aligned}
& \mathbf{R}_{1}\left(\theta_{1}\right)=\left(\begin{array}{ccc}
1 & 0 & 0 \\
0 & \cos \theta_{1} & -\sin \theta_{1} \\
0 & \sin \theta_{1} & \cos \theta_{1}
\end{array}\right), \\
& \mathbf{R}_{2}\left(\theta_{2}\right)=\left(\begin{array}{ccc}
\cos \theta_{2} & 0 & \sin \theta_{2} \\
0 & 1 & 0 \\
-\sin \theta_{2} & 0 & \cos \theta_{2}
\end{array}\right), \\
& \mathbf{R}_{3}\left(\theta_{3}\right)=\left(\begin{array}{ccc}
\cos \theta_{3} & -\sin \theta_{3} & 0 \\
\sin \theta_{3} & \cos \theta_{3} & 0 \\
0 & 0 & 1
\end{array}\right)
\end{aligned}
$$

$\mathbf{R}$ is also the passive transformation from the body to the global coordinates. Therefore, the Euler angle derivatives are found as $[6,7]$

$$
\begin{aligned}
& \dot{\theta}_{1}=\omega_{1}+\left(\omega_{2} \sin \theta_{1}+\omega_{3} \cos \theta_{1}\right) \tan \theta_{2} \\
& \dot{\theta}_{2}=\omega_{2} \cos \theta_{1}=\omega_{3} \sin \theta_{1} \\
& \dot{\theta}_{3}=\frac{\omega_{2} \sin \theta_{1}+\omega_{3} \cos \theta_{1}}{\cos \theta_{2}}
\end{aligned}
$$

Euler angles are used to rotate the local mass centre velocity into globals, which are then integrated to find the global $x, y, z$ coordinates of $G$ (vehicle centre of gravity). Vehicle accelerations are also found in both local and global coordinates, but only for post-processing purposes.

\subsection{Suspension and steering}

Nominal suspension deflections and velocities are found (nominal because bump and rebound stop forces are ignored in this analysis). This is non-trivial because of the largeangle formulation highlighted here. There are three stages (see Fig. 2).

1. Find $P$, the nominal contact patch centre that translates and rotates with the vehicle body - based on the static 'trim' condition of the body, including static tyre deflection. Using the mass centre $G$ as a reference point

$$
\boldsymbol{r}_{P}=\boldsymbol{r}_{G}+\mathbf{R}^{\{\mathrm{B} \rightarrow \mathrm{G}\}} \boldsymbol{r}_{P}^{\{\mathrm{B}\}}
$$

where the curly bracket superscripts denote the coordinate system used: $\boldsymbol{r}_{P}^{\{\mathrm{B}\}}$ is the position of the nominal contact patch centre in the vehicle body coordinates, based at $G$, and $\mathbf{R}^{\{\mathrm{B} \rightarrow \mathrm{G}\}}$ is the (passive) rotation matrix that converts from the body to the global coordinates using the Euler angles. In the remainder of this section it is assumed that similar transformations into globals have been carried out as necessary.

2. Find $Q$, the new position of $P$ obtained by translating $Q$ in body $z$ axes (no account is taken here of the suspension geometry at this point, to include scrub effects, etc., as this has a negligible effect on the suspension vertical travel). This defines the nominal suspension deflection.

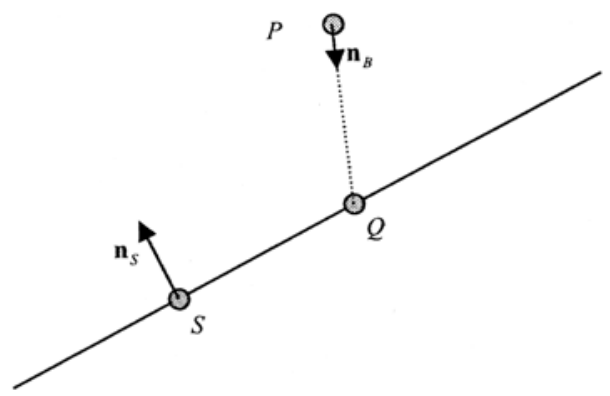

Fig. 2 Representation of the point at the centre of the contact patch 
Except where the wheel is out of contact with the road, the distance between $P$ and $Q$ can be expected to be small compared with the typical wavelength of the surface. If the surface is defined by $z=f(x, y)$, an initial approximation to $Q$ is given by

$$
\boldsymbol{r}_{S}=\left[x_{P}, y_{P}, f\left(x_{P}, y_{P}\right)\right]^{\mathrm{T}}
$$

The approximation will be poor unless both the vehicle and the surface are considered to be close to horizontal. $S$ will be close in distance to the required point, so an improved approximation can be found by a planar representation of the road surface around $S$.

This is defined by $\boldsymbol{n}_{S}$, which is the unit (upward) normal to the road surface at $S$

$$
\left(\boldsymbol{r}-\boldsymbol{r}_{S}\right) \cdot \boldsymbol{n}_{S}=0
$$

Since $Q$ is obtained by translating $P$ parallel to the body $z$ unit vector $\boldsymbol{n}_{\mathrm{B}}$, then

$$
\boldsymbol{r}_{Q}=\boldsymbol{r}_{P}+\lambda \boldsymbol{n}_{B}
$$

Here, $\lambda$ is the sum of the suspension and tyre deflections (relative to the static equilibrium position, ignoring the actions of bump or rebound stops) and may be found by solving the above two equations to give

$$
\lambda=\frac{\left(\boldsymbol{r}_{S}-\boldsymbol{r}_{P}\right) \cdot \boldsymbol{n}_{S}}{\boldsymbol{n}_{\mathrm{B}} \cdot \boldsymbol{n}_{S}}
$$

In the model this is calculated in the global coordinates. Note that the estimation of suspension deflection can be refined via an iteration process on the choice of local surface normal, and, by including the suspension geometry effects, the extra computational load is not justified.

3. Analyse the velocity of $Q$ to determine the suspension velocity, and hence the overall velocity vector of the contact patch. As $Q$ moves on the surface, its velocity is based on the rigid body motion of the vehicle, except for the addition of suspension velocity

$$
v_{Q}=v_{G}+\omega \times\left(r_{Q}-r_{G}\right)+\mu n_{\mathrm{B}}
$$

where $\mu$ is the (expansion) velocity of the suspensiontyre combination. Since $Q$ is moving within the plane, $\boldsymbol{v}_{Q} \cdot \boldsymbol{n}_{S}=0$ and hence

$$
\mu=\frac{\boldsymbol{n}_{S} \cdot\left(\boldsymbol{v}_{G}+\omega \times\left(\boldsymbol{r}_{Q}-\boldsymbol{r}_{G}\right)\right)}{\boldsymbol{n}_{S} \cdot \boldsymbol{n}_{\mathrm{B}}}
$$

Tyre vertical compliance is included in the suspension model. The unsprung mass is considered to be included in with the vehicle body, so the 'massless' wheel constitutes a 'half degree of freedom', involving one state variable: the suspension deflection. In outline this works as follows: as above, the combined tyre/ suspension displacement and velocities are known. The suspension deflection state, $z_{\mathrm{s}}$, is used to determine the tyre deflection as $z_{\mathrm{t}}=\lambda-z_{\mathrm{s}}$, and both 'spring' forces acting on the wheel (see Fig. 3) are known. After taking into account the geometry of the system and the in-plane forces, this assumption implies a required damper force, and (via an inverse damper map) the required suspension velocity is used to update the suspension deflection state.

Limits on tyre and suspension travel are implemented as simple modifications to the above

$$
z_{\mathrm{t}}^{\min } \leqslant z_{\mathrm{t}} \leqslant z_{\mathrm{t}}^{\max }, \quad z_{\mathrm{s}}^{\min } \leqslant z_{\mathrm{s}} \leqslant z_{\mathrm{s}}^{\max }
$$

Here, $z_{\mathrm{t}}^{\text {min }}$ represents the maximum tyre compression and a nominal maximum 'rim contact' tyre force $F_{\mathrm{t}}^{\max }$ is applied. Alternatively, $z_{\mathrm{t}}^{\min }$ represents loss of tyre contact, where $F_{\mathrm{t}} \rightarrow 0$.

When suspension end-stops are exceeded, the damper force is 'overridden' by virtual bump-stops; the calculated velocity is modified to prevent an excursion beyond the workspace limits as

$$
\begin{aligned}
& \dot{z}_{\mathrm{S}}=\max \left\{\dot{z}_{\mathrm{S}}^{\text {calc }}, \dot{z}_{\mathrm{S}}^{\text {small }}\right\} \quad \text { if } z_{\mathrm{S}}<z_{\mathrm{S}}^{\min } \\
& \dot{z}_{\mathrm{S}}=\min \left\{\dot{z}_{\mathrm{S}}^{\text {calc }},-\dot{z}_{\mathrm{S}}^{\text {small }}\right\} \quad \text { if } z_{\mathrm{S}}>z_{\mathrm{S}}^{\max }
\end{aligned}
$$

Now, turning to the suspension geometry effects, such as anti-dive characteristics and scrub effects, the balance is obtained via application of the principle of virtual work in the vehicle body coordinates. Considering the active forces and moments acting on the wheel/hub assembly when the body is fixed (see Fig. 4), the virtual work takes the form

$$
F_{x} \delta x+F_{y} \delta y+F_{z} \delta z+F_{\mathrm{s}}(-\delta z)+T_{\mathrm{d}} \delta v=0
$$

Here, all the forces are acting on the wheel/hub assembly, and link reaction forces (ball-joints at the body connections) make no contribution. Force $F_{z}$ increases with tyre extension but carries a large negative component owing to the

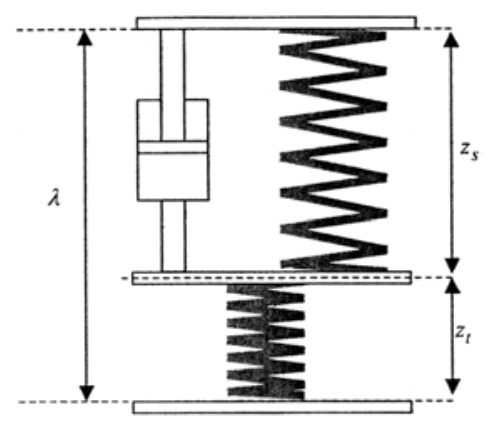

Fig. 3 Tyre and suspension travel 


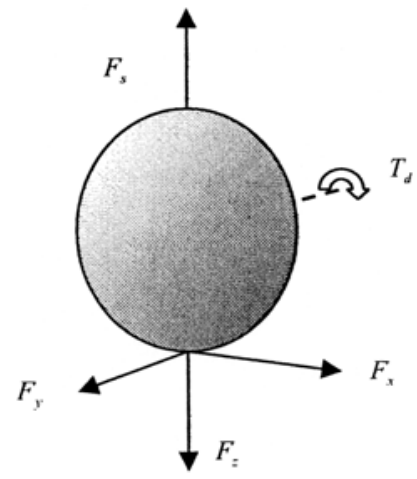

Fig. 4 Forces and moments for the calculation of virtual work

static load; overall it is negative, tending to zero as the tyre lifts off the road surface. Similarly, $F_{\mathrm{s}}$ would usually be negative but increases as the suspension is expanded. The virtual work equation is based on the body-fixed coordinates and $z$ is the suspension deflection (vertical height change of the contact patch centre) and is considered as an independent variable. As the suspension is deflected, $\delta x$ and $\delta y$ (contact patch forward progression and lateral scrub respectively) follow from mapping the suspension geometry as

$$
\delta x=\left(\frac{\mathrm{d} x}{\mathrm{~d} y}\right) \delta z, \quad \delta y=\left(\frac{\mathrm{d} y}{\mathrm{~d} z}\right) \delta z
$$

where $F_{\mathrm{s}}$ is the net suspension force, based on the vertical wheel travel. If the spring or damper is not directly aligned with the wheel vertical motion (as is typically the case), then the principle of virtual work can be used again to obtain $F_{\mathrm{s}}(z)$ for example, if $s$ is the spring deflection and $\tilde{F}_{\mathrm{s}}(s)$ is the variation in the component of spring with deflection, then

$$
F_{\mathrm{s}}(z)=\tilde{F}_{\mathrm{s}}(s) \frac{\mathrm{d} s}{\mathrm{~d} z}
$$

In the virtual work equation, $T_{\mathrm{d}}$ is the drive torque (assumed to be generated from an inboard differential) and $\delta v$ is the change in the caster angle. Brake torques do not contribute, because they are considered as internal to the wheel-hub assembly.

The virtual work equation can be written (in the body coordinates) as

$$
F_{x} d_{x}+F_{y} d_{y}+F_{z}-F_{\mathrm{s}}+T_{\mathrm{d}} d_{v}=0
$$

where $d_{x}=(\mathrm{d} x / \mathrm{d} z)$ etc. Then, defining

$$
\boldsymbol{d}=\left[d_{x} d_{y} 1\right]^{\mathrm{T}}
$$

the virtual work equation becomes

$$
\boldsymbol{F} \cdot \boldsymbol{d}=F_{\mathrm{s}}-T_{\mathrm{d}} d_{v}
$$

This must now be transformed to the 'tyre' coordinates in order to find the unknown road normal force. Leaving aside the details for now, let $\mathbf{R}^{\{\mathrm{B} \rightarrow \mathrm{T}\}}$ be the (passive) rotation matrix that transforms vector components from the body-fixed axes to the tyre axes. The dot product is the same in any coordinate system, thus transforming to tyre coordinates as

$$
\boldsymbol{F}^{\{\mathrm{T}\}}=\mathbf{R}^{\{\mathrm{B} \rightarrow \mathrm{T}\}} \boldsymbol{F}^{\{\mathrm{B}\}}, \quad \boldsymbol{d}^{\{\mathrm{T}\}}=\mathbf{R}^{\{\mathrm{B} \rightarrow \mathrm{T}\}} \boldsymbol{d}^{\{\mathrm{B}\}}
$$

Making use of equation (27) yields

$$
F_{\mathrm{s}}=F_{x}^{\{\mathrm{T}\}} d_{x}^{\{\mathrm{T}\}}+F_{y}^{\{\mathrm{T}\}} d_{y}^{\{\mathrm{T}\}}+F_{z}^{\{\mathrm{T}\}} d_{z}^{\{\mathrm{T}\}}+T_{\mathrm{d}} d_{v}=0
$$

With $F_{z}{ }^{\{\mathrm{T}\}}$ known from the tyre deflection and $F_{x}^{\{\mathrm{T}\}}$ and $F_{y}^{\{\mathrm{T}\}}$ obtained as output from the tyre model, this determines the body vertical suspension force, $F_{\mathrm{s}}$. Subtracting the spring component (including static load) and inverting the damper map gives the suspension velocity as required above.

The transformation from body to tyre coordinates is now derived. In order to account for steering angle, the steering axis geometry, toe, camber and caster change. The Euler angles and road normal are also needed, because the tyre $Z$ axis is normal to the road. Consider a general rotation through angle $\phi$ about an axis defined by a unit vector $\boldsymbol{n}$. As an 'active' rotation, an arbitrary vector $\boldsymbol{v}$ is rotated and the coordinates are fixed, so $\boldsymbol{v} \rightarrow \boldsymbol{v}^{\prime}$, with

$$
\boldsymbol{v}^{\prime}=(\boldsymbol{v} \cdot \boldsymbol{n}) \boldsymbol{n}(1-\cos \phi)+\boldsymbol{v} \cos \phi+(\boldsymbol{n} \times \boldsymbol{v}) \sin \phi
$$

Therefore, for steering rotation about the kingpin axis, for example, for the right front wheel

$$
\boldsymbol{n}=\left(\begin{array}{c}
\cos \gamma \sin v \\
\sin \gamma \\
\cos \gamma \sin v
\end{array}\right)
$$

This is a unit vector pointing along the kingpin axis ( $v=$ caster angle, $\gamma=$ lateral inclination angle), and

$$
\phi=\delta_{\mathrm{k}}\left(z_{\mathrm{sus}}\right)+\delta
$$

This is the actual steer angle plus a kinematics term $\delta_{\mathrm{k}}\left(z_{\text {sus }}\right)$ which accounts for bump-steer and the static toe angle. In equation (30), $v$ is a unit vector normal to the wheel plane, and it is assumed that, starting from the reference (trim) condition, the suspension is deflected first, inducing bump-camber and bump-steer (these angles are small, so the rotation sequence is unimportant and it is convenient to effect the camber first), then rotated by angle $\delta$ about the kingpin axis (see Fig. 5). Note that both caster and lateral inclination are considered constant in this model but can easily be mapped as functions of suspension travel if required. 


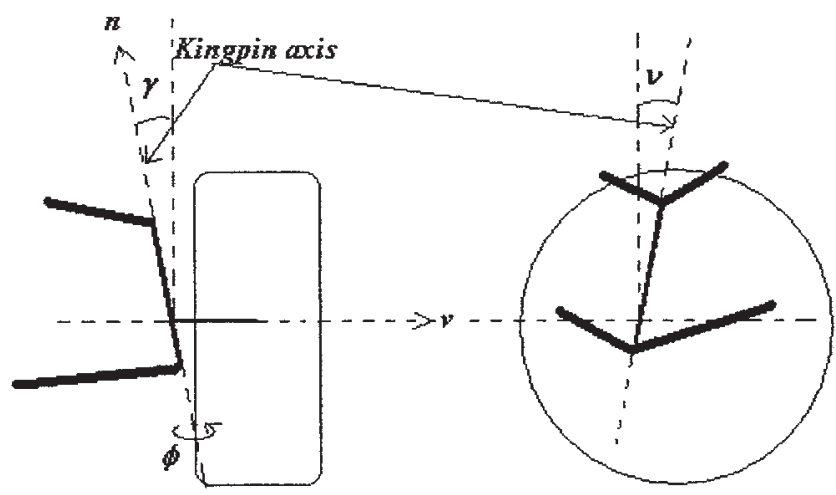

Fig. 5 Virtual steering axis

The overall rotation of the wheel plane normal using the body-fixed axes is

$$
\left(\begin{array}{l}
0 \\
1 \\
0
\end{array}\right) \rightarrow\left(\begin{array}{c}
0 \\
\cos \rho \\
\sin \rho
\end{array}\right)=\boldsymbol{v} \rightarrow \boldsymbol{v}^{\prime}
$$

Working fully in the body-fixed coordinates requires the transformation of the road surface normal into the body coordinates in the following form

$$
\boldsymbol{n}_{S}=\boldsymbol{n}_{S}^{\{\mathrm{B}\}}=\boldsymbol{R}^{\{\mathrm{G} \rightarrow \mathrm{B}\}} \boldsymbol{n}_{S}^{\{\mathrm{G}\}}
$$

Removing the road normal component from $\boldsymbol{v}^{\prime}$ and rescaling gives the y axis base vector for the tyre coordinate system

$$
\boldsymbol{e}_{2}=\frac{\boldsymbol{v}^{\prime}-\left(\boldsymbol{v}^{\prime} \cdot \boldsymbol{n}_{S}\right) \boldsymbol{n}_{S}}{\left|\boldsymbol{v}^{\prime}-\left(\boldsymbol{v}^{\prime} \cdot \boldsymbol{n}_{S}\right) \boldsymbol{n}_{S}\right|}
$$

The $z$ axis vector is simply the road surface normal, $\boldsymbol{e}_{3}=\boldsymbol{n}_{S}$, and the $x$ axis vector follows from the cross-product

$$
\boldsymbol{e}_{1}=\boldsymbol{e}_{2} \times \boldsymbol{e}_{3}
$$

If a vector $\boldsymbol{a}=\left[\begin{array}{lll}a_{1} & a_{2} & a_{3}\end{array}\right]^{\mathrm{T}}$ is given in the body-fixed coordinates and is multiplied by the matrix $\mathbf{R}=\left[\boldsymbol{e}_{1} \boldsymbol{e}_{2} \boldsymbol{e}_{3}\right]$, formed from components of $\boldsymbol{e}$ (all in the body coordinates), this yields

$$
\boldsymbol{a}^{\prime}=a_{1} \boldsymbol{e}_{1}+a_{2} \boldsymbol{e}_{2}+a_{3} \boldsymbol{e}_{3}
$$

which is the vector 'actively' transformed from the body to the tyre axes. Hence, $\mathbf{R}$ is also the passive rotation matrix from the tyre coordinates to the body coordinates

$$
\mathbf{R}^{\{\mathrm{T} \rightarrow \mathrm{B}\}}=\left[e_{1} \boldsymbol{e}_{2} \boldsymbol{e}_{3}\right]
$$

For the left front wheel the above analysis is the same but the sign of $\lambda$ is essentially reversed. If symmetry is assumed, the look-up tables for $\delta_{\mathrm{k}}\left(z_{\text {sus }}\right)$ and $\rho\left(z_{\text {sus }}\right)$ must also have negative signs applied, and the model allows for independent left-right suspension geometry. For the rear wheels the formulation is the same: typically, the commanded steer is zero, and the caster and lateral inclination angles are assumed to be zero.

This essentially completes the suspension and steering analysis. It is noteworthy that the model is not currently set up to include steering torque output. The above steering geometry allows steering torque to be found quite simply via the inclusion of the mapped location $\boldsymbol{r}_{\mathrm{A}}\left(z_{\text {sus }}\right)$ of a reference point on the kingpin axis in the body-fixed coordinates (e.g. the outer ball joint on the upper A-arm, or a body-fixed upper mount on a MacPherson strut).

\subsection{Driveline and tyres}

These deal with the wheel spin dynamics (four states) and a series of first-order lags (with fixed time constants) for the build-up of engine torque (one state), braking torques (four states) and in-plane tyre forces (eight states); overall there are 17 states. The tyre $x$ and $y$ components of velocity of the extended vehicle body, at the contact patches, including roll, pitch, etc., are used to find the longitudinal and lateral slip ratios. These are fed into the tyre model to obtain 'prefiltered' tyre forces, $F_{\mathrm{p}}$, which are lagged in the generation of the actual tyre forces, $F_{\mathrm{a}}$. This is schematically shown in Fig 6.

Subsequently, force/torque balance across the wheels determines the wheel acceleration and the wheel speeds. To prevent excessive wheel spin and the associated numerical integration problem, some additional non-linear damping is added to limit the maximum wheel accelerations. Optional simplified ABS/TCS functionality is also included to reduce the brake and drive torque demands when preset slip limits are exceeded.

\subsection{Driver model}

There is a choice of closed-loop [8] or open-loop driver models. The closed-loop driver depends on a reference vector field of target directions and speeds, which couples to simple proportional-integral (PI) controllers for both steering and speed control. The vector field 'solves' the path and speed planning aspects of the driving task. An Ackerman steer provides a simple 'model' input for steering control, and the remainder of the steering control is via PI feedback compensation. Tracking to the speed reference

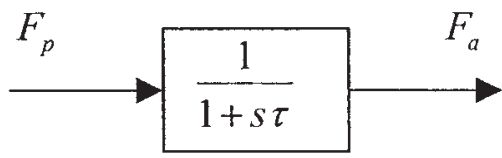

Fig. 6 Lag in tyre forces 
control is entirely via the PI feedback. In more detail, for speed control forward velocity is the key parameter as

$$
y_{1}=-\underbrace{\mathrm{KI}_{1} \int \varepsilon_{1}}_{\text {integral }}-\underbrace{\mathrm{KP}_{1} \varepsilon_{1}}_{\text {proportional }}
$$

Deviation $\varepsilon_{1}=\left(S-S_{\text {ref }}\right)$

where the deviation $\varepsilon_{1}$ of the actual speed from its desired value determines whether the output of the system would provide acceleration or a braking command. The output of the system $y_{1}$ consists of two elements, integral and proportional elements, where the integral gain is $\mathrm{KI}$ and the proportional gain is $\mathrm{KP}$.

The same approach is used for directional control, in which case a three-element model of velocity is required: the longitudinal, lateral and yaw components of velocity. For directional control, $\theta_{\mathrm{v}}$, which is the angle (in the global coordinates) of the reference vector field vector, $\beta$ is the yaw angle of the car, so $\varepsilon_{2}$ represents a directional error between where the car is pointing and where it should be heading

$$
\text { Deviation } \varepsilon_{2}=\left(\beta-\theta_{\mathrm{v}}\right)
$$

$$
\text { Steering angle command } y_{2}=-\underbrace{\mathrm{KI}_{2} \int \varepsilon_{2}}_{\text {integral }}-\underbrace{\mathrm{KP}_{2} \varepsilon_{2}}_{\text {proportional }}
$$

The 'open-loop' driver is specified by desired steer angle and vehicle speed time histories, and once again the speed control is feedback based. However, since the desired speed is precomputed, a desired acceleration time history is derived to provide an approximate input into the vehicle (equivalent torque demand), which is corrected by the PI feedback.

The entire intermediate model described above was created in the environment of Matlab/Simulink.

\section{EXPERIMENTAL AND SIMULATION RESULTS}

In order to validate the above model it was necessary to compare it with the actual vehicle data. For this purpose, five different types of test were conducted. These included:

(a) constant acceleration of $0.2 \mathrm{~g}$ with an initial speed of $10 \mathrm{~km} / \mathrm{h}$

(b) constant deceleration of $0.5 \mathrm{~g}$ with an initial speed of $60 \mathrm{~km} / \mathrm{h}$

(c) speed bump analysis - constant speed $(10 \mathrm{~km} / \mathrm{h})$ throughout negotiation of the speed bump;

(d) speed bump analysis-constant speed $(20 \mathrm{~km} / \mathrm{h})$ throughout traversal of the speed bump;

(e) speed bump analysis - the initial speed of $30 \mathrm{~km} / \mathrm{h}$ is given a deceleration of $0.15 \mathrm{~g}$ before the vehicle negotiates the speed bump.

\subsection{Experimental procedure}

A standard D class passenger car is used. The tests were actual road manoeuvres as this is the most representative of vehicle performance, rather than the usual chassis dynamometer tests where the full effect of vehicle inertia under various motions, particularly in combined bounce and pitch dynamics, cannot be realized. The sensors are placed at four places to monitor longitudinal acceleration, body bounce, wheel vertical acceleration and suspension deflection, as depicted in Fig. 7. For the purpose of model validation, the vertical displacement of the suspension system is more important and is discussed here.

The employed sensors are:

Sensor A-vertical accelerometer

Sensor B-longitudinal accelerometer

Sensor C-wheel accelerometer

Sensor D-suspension deflection sensor (an LVDT)

The type of accelerometer used is a Setra, model 141 accelerometer with a $\pm 2 g$ range for longitudinal measurements and a $\pm 15 g$ range for the case of vertical measurements.
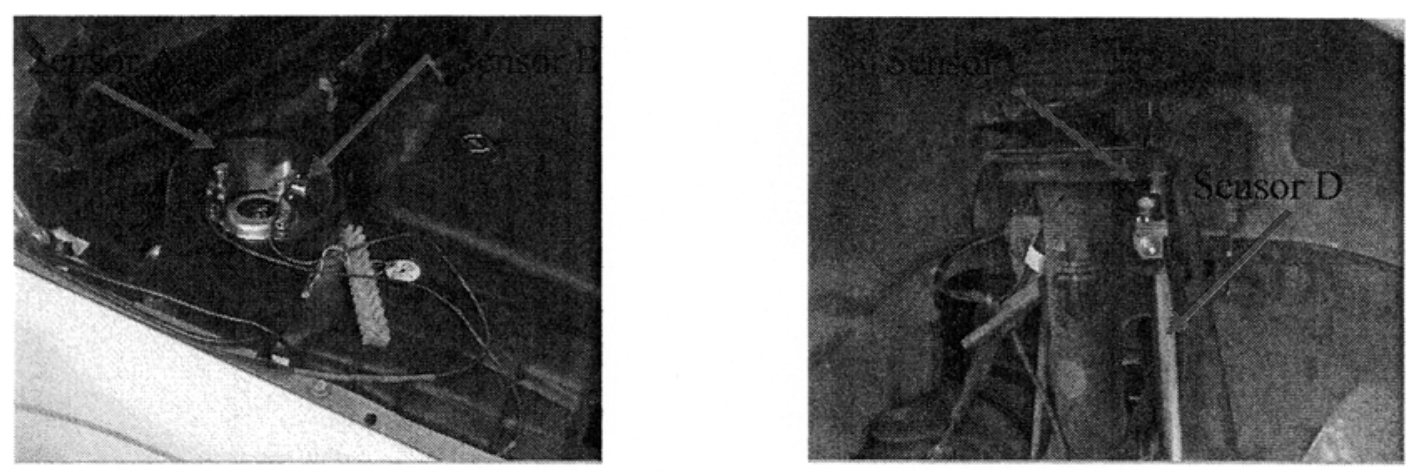

Fig. 7 Sensor locations 


\subsection{Experimental results and theoretical predictions}

For the multi-body model, two types of suspension characteristic are considered, one without anti-squat and anti-dive features and the other with these characteristics provided by the vehicle manufacturer. The current model uses linear damping and does not include camber changes, kingpin inclination and bump-steer effect. These features can be included in the future developments of the reported model for better representation of the suspension system. An open-loop driver model is used.

\subsubsection{Test 1: constant acceleration of $0.2 \mathrm{~g}$ with an initial speed of $10 \mathrm{~km} / \mathrm{h}$}

Simulation models cannot exactly replicate the behaviour of a vehicle in accelerated motion. This is because the rate of change is driver dependent and in a simulation exercise is usually considered to be ideally instantaneous. Figure 8 shows this difference. Owing to the step change caused by the instantaneous application of throttle, the simulation results tend to exhibit an initial rapid oscillatory behaviour. Nevertheless, the conformity of model predictions to experimental findings is remarkably good after this initial anomaly.

To observe the effectiveness of the anti-squat and antidive features, it is necessary to gauge vehicle performance, when accelerated from coasting to drive condition (as shown in Fig. 8), or in hard braking from coasting. The result for the former case is shown in Fig. 9 for the front suspension in this rear wheel drive vehicle. There are three curves, one of which depicts the actual road data for suspension vertical deflection while the other two correspond to numerical predictions: one for the suspension model without the rear leading anti-squat arms and the other with this feature included in the model. It can be observed that the

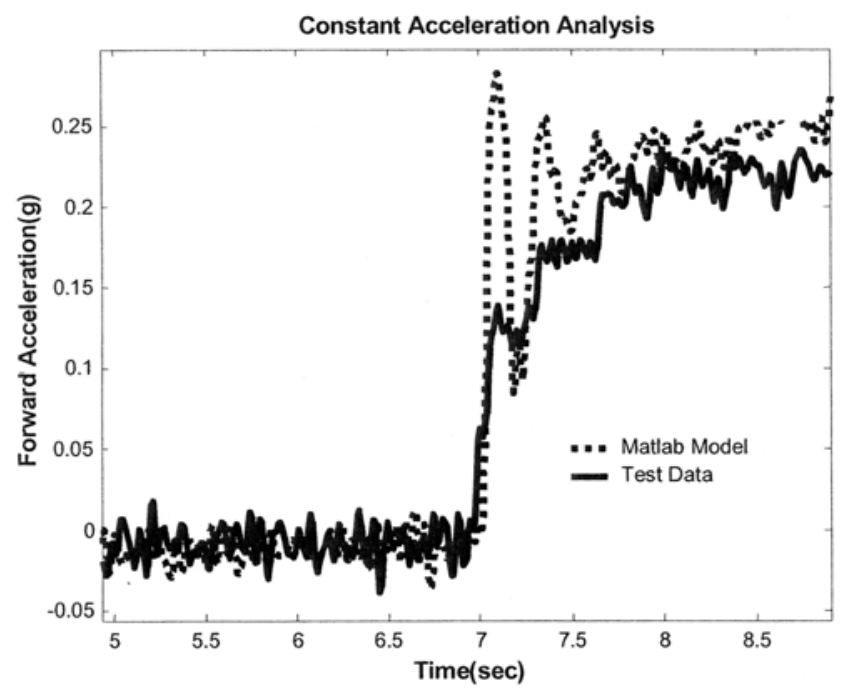

Fig. 8 Forward acceleration

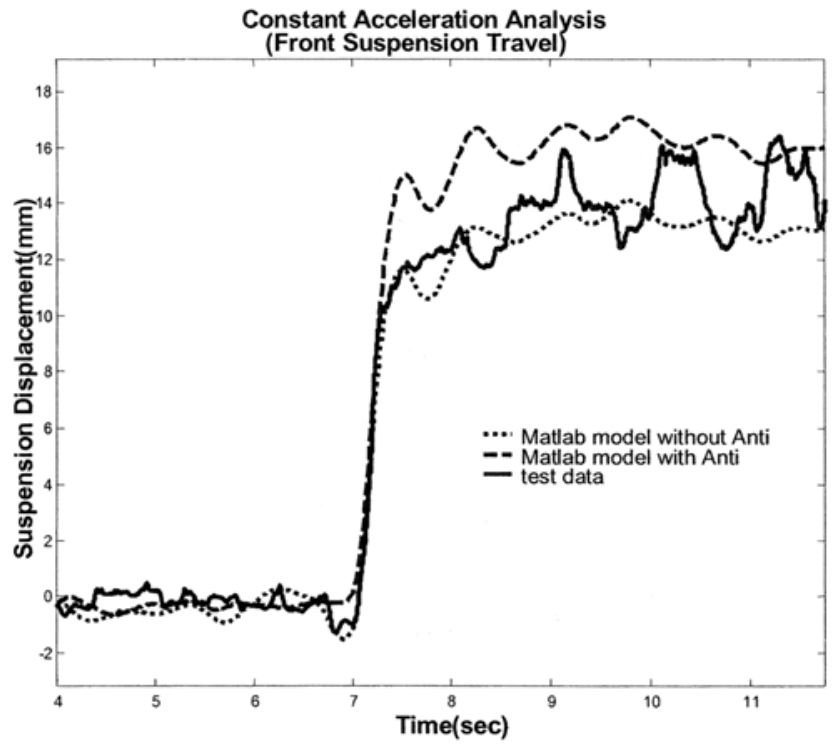

Fig. 9 Front suspension deflection

experimental results fall in between the two sets of numerical predictions. This is because, with the leading arms, the rear suspension deflects less, and consequently the front suspension carries a greater proportion of the inertial force than would be expected. When the leading anti-squat arms are removed, weight transfer to the rear under acceleration takes place, as expected, and the front suspension vertical travel is reduced. None of the predicted results totally conforms to the experiment. There are two possible reasons.

1. The anti-squat arm is considered without joint compliance which would yield higher stiffness than that actually existing in the vehicle (therefore less rear squat and lower weight transfer to there).

2. Suspension deflection occurs in the real situation in both vertical and horizontal directions, together with the angular movement of the control arms as the result of friction torque transfer. The models do not directly obtain these. The predictions do, however, give fairly good indications of vehicle dynamics.

\subsubsection{Test 2: constant deceleration of $0.5 \mathrm{~g}$ with an initial speed of $60 \mathrm{~km} / \mathrm{h}$}

A case of hard braking typical of an emergency stop was investigated. In such cases a constant deceleration of $0.5 \mathrm{~g}$ may be considered as typical. However, in reality, the driver does not maintain a constant brake pressure, because under dive conditions the seating posture alters and consequently there is some gradual loss of pedal brake force. This can be observed in the experimental trace of Fig. 10 and accounts for the difference in the final portion of deceleration in the figure. Elsewhere, very good agreement is observed between theory and experiment.

When considering vertical front suspension travel under hard braking conditions (see Fig. 11), an opposite effect to 


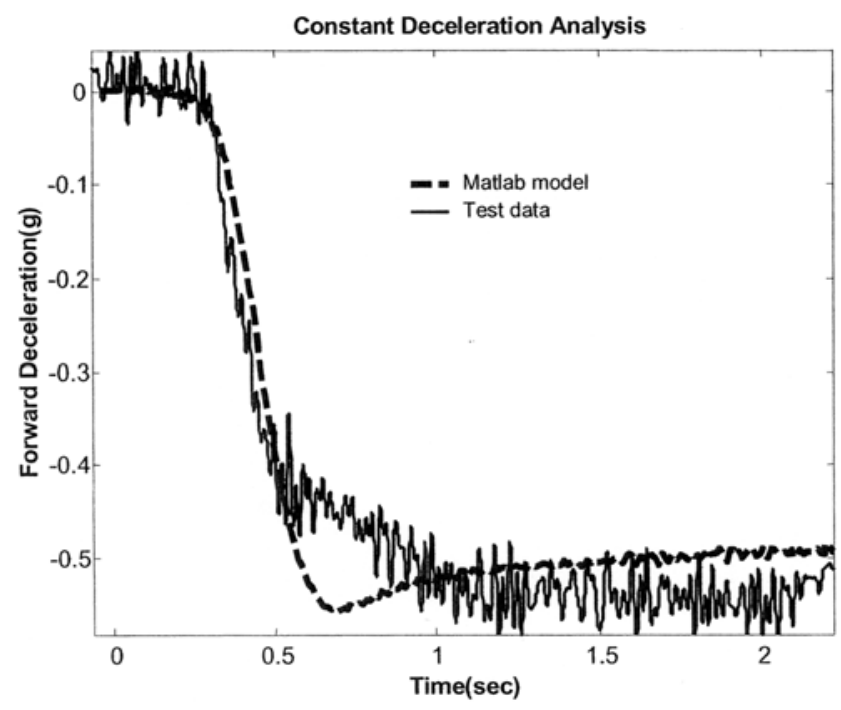

Fig. 10 Forward deceleration

that of Fig. 9 is observed, as would be expected. In this case, the semi-trailing anti-dive arms resist inertial load transfer to the front of the vehicle. Therefore, the suspension deflection is less than that predicted without this feature, which conforms closer to the actual vehicle data. The discrepancy is due to the effects of other resisting elements such as suspension arm bushings and joints which are not included in the simple suspension model described previously. Another omission in the simulation model is the damping behaviour of the suspension arm and trailing arm bushing mounts, this being the reason for the lightly damped oscillatory characteristics of both the numerical traces when compared with

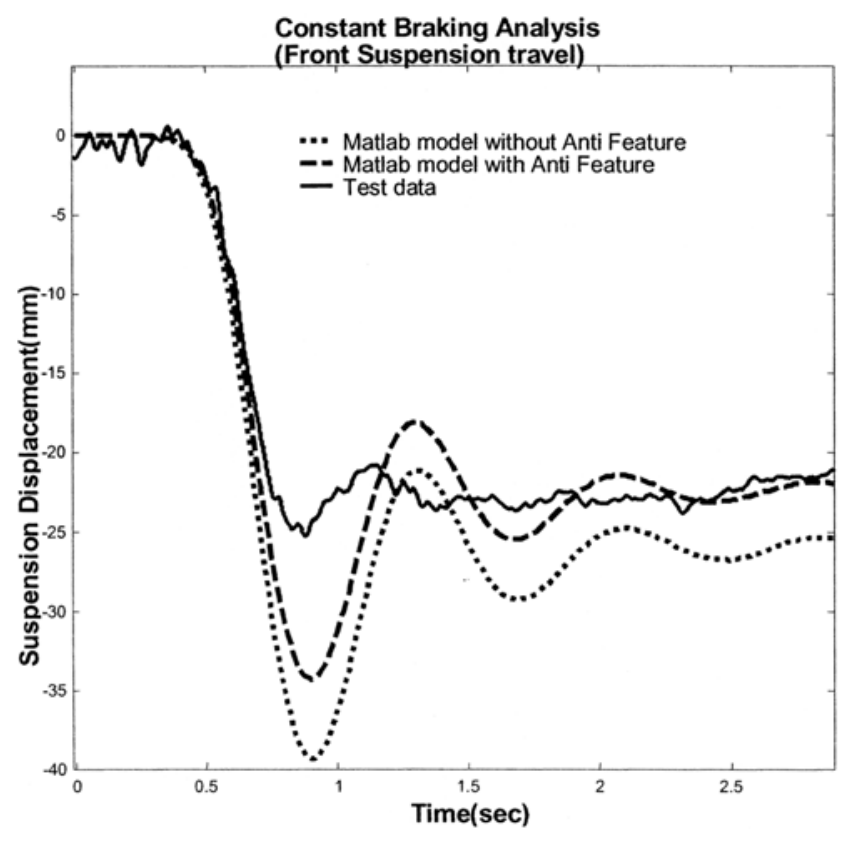

Fig. 11 Front suspension deflection the experimental curve in the same figure. Note, however, that, owing to the generally underdamped nature of the bushings, the frequency of oscillation is almost the same in both cases. The results of the tests give an indication of vehicle pitch plane dynamics. Another important consideration is the combined effects of vehicle bounce and pitch motions, increasingly encountered in today's roads where traffic calming measures invariably involve the use of speed bumps. Ride comfort and handling, traditionally kept apart in analysis work, combine in importance under such manouevres.

\subsubsection{Test 3: speed bump analysis—constant speed $(10 \mathrm{~km} / \mathrm{h})$ throughout negotiation of the speed bump}

Thus far the results presented are for accelerated motion which in an ideal sense corresponds to vehicle pitch plane dynamics. However, the vehicle body is often subject to combined pitch, bounce and roll. In a straight-line motion with both wheels going over a low-height barrier, the effect of roll is diminished, but the individual contributions of pitch and bounce cannot be isolated owing to the coupled nature of the dynamics.

The first combined bounce and pitch dynamics test corresponds to negotiating a speed bump of $4 \mathrm{~m}$ length and $110 \mathrm{~mm}$ height with a constant velocity of $10 \mathrm{~km} / \mathrm{h}$. Figure 12 shows the monitored experimental data and the corresponding numerical predictions with and without anti-squat and anti-dive features. All the traces show much more complex motions than the previous pitch plane dynamics cases owing to the combined effect of this motion with vertical bounce of the vehicle. The time taken at a constant speed of $10 \mathrm{~km} / \mathrm{h}$ to traverse the bump

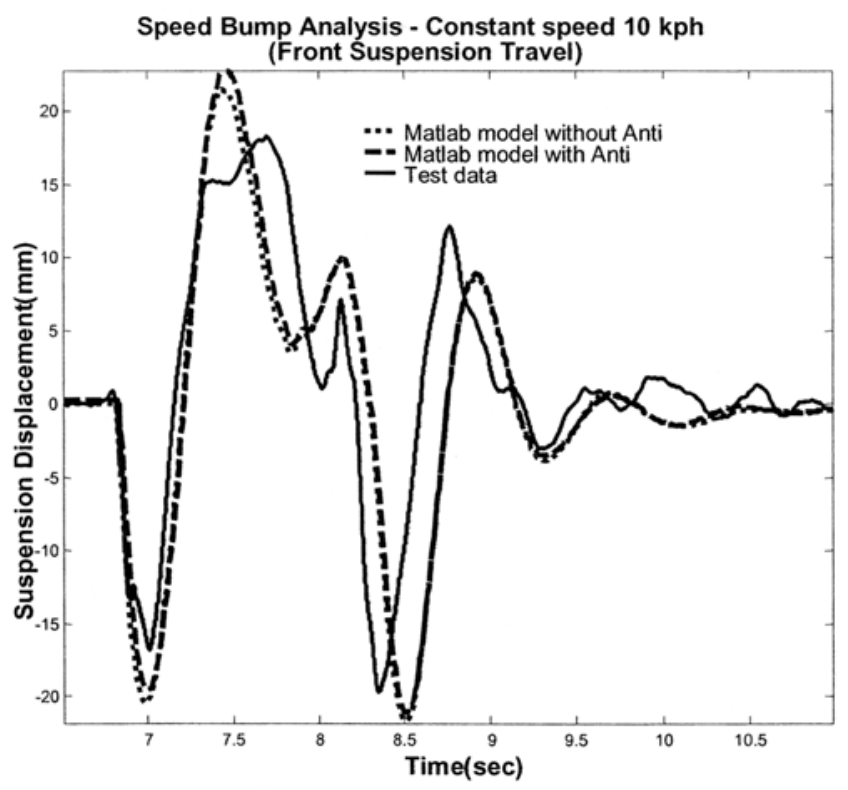

Fig. 12 Front suspension deflection with bounce and pitch dynamics 
is approximately $1.5 \mathrm{~s}$. The front wheels reach the bump at $7 \mathrm{~s}$ after the commencement of the simulation or road test, and finally the rear wheels leave the bump at $t=8.5 \mathrm{~s}$ (as shown in the figure). Following either of the three traces (and note that both the numerical results almost coincide with each other), the front suspension travel initially undergoes an upward deflection (referred to as jounce), followed by a return travel and rebound (an extended geometry due to off-loading) as the front axle begins to fall off the bump. This reaches a maximum float of front suspension (indicated by the maximum positive deflection at around $t=7.5 \mathrm{~s}$ ). As the front wheels fall off the bump, the extended (floating) suspension begins to return to its equilibrium position, while the rear wheels climb onto the bump, momentarily carrying the major inertial load, and hence resulting in the second less pronounced maximum in the vertical front suspension travel. As the rear wheels reach the summit of the bump, maximum load transfer to the front occurs, resulting in maximum deflection (the second minima in any of the traces in the figure). This is combined with the impact of the front wheels onto the flat surface of the road. The load almost instantaneously transfers to the rear thereafter, and, after a few small oscillations, steady conditions are reached, with the vehicle being on the flat road.

All the traces follow the same pattern and are reasonably in accord with each other. The horizontal shifts in time between the theoretical and experimental results are due to omission of damping and non-linearity effects in the former case, such as the elastokinetic effects in real suspension systems caused by structural compliance. The lack of a significant difference between the numerical results with and without anti-dive and anti-squat characteristics is due to lack of sufficient time for leading and trailing arms to influence the vehicle dynamics, and in particular these features have less effect with prounced vehicle bounce. This point can be corroborated by further decrease in any differences in the numerical results with increasing vehicle speed.

\subsubsection{Test 4: speed bump analysis-constant speed $(20 \mathrm{~km} / \mathrm{h})$ throughout traversal of the speed bump}

For this purpose the speed of the vehicle was doubled to $20 \mathrm{~km} / \mathrm{h}$ and kept constant while negotiating the bump. Monitoring the front suspension vertical travel, shown in Fig. 13, indicates the same pattern of variation as in the previous case, with the exception that much greater deflection and extension behaviour is observed, this being due to increased inertial force and higher impact forces at the tyres, transmitted to the suspension elements. The effect of bounce motion has also become more dominant owing to these increased vertical forces, as a result of which the influence of anti-squat and anti-dive features has all but disappeared. This results in almost coincident alternative numerical predictions and a closer fit with the experimental results.

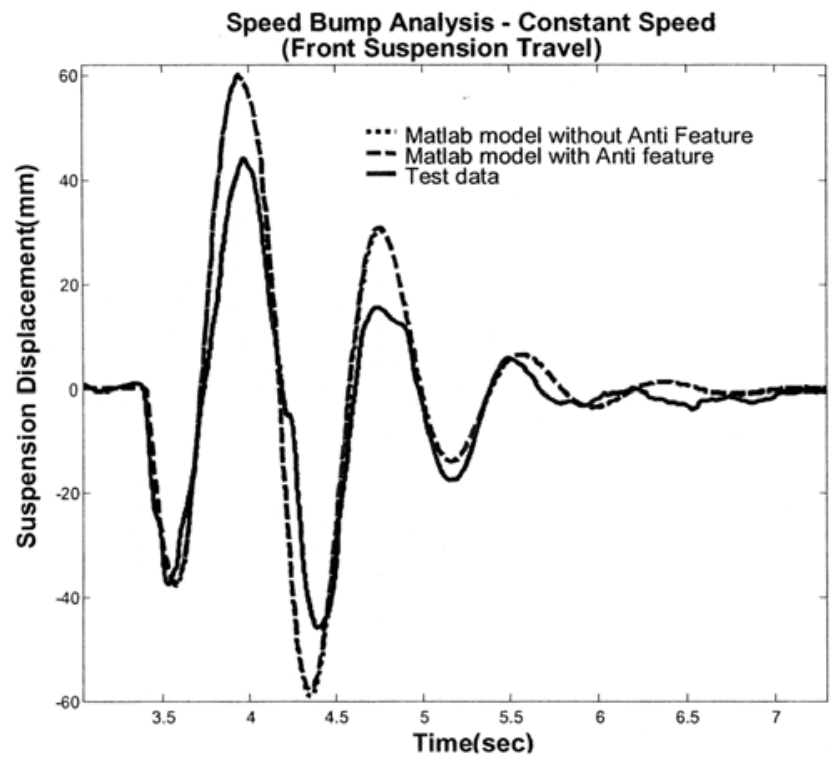

Fig. 13 Front suspension deflection

\subsubsection{Test 5: Speed bump analysis—the initial speed of $30 \mathrm{~km} / \mathrm{h}$ is given a deceleration of $0.15 \mathrm{~g}$ before the vehicle negotiates the speed bump}

A prior braking action, however, usually accompanies negotiation of a speed bump. This represents a more realistic scenario, particularly at a higher initial velocity, in this case at $30 \mathrm{~km} / \mathrm{h}$. Figure 14 shows the front suspension behaviour under this condition for all three alternatives as in the previous figures. A deceleration of $0.15 \mathrm{~g}$ is typical of such a braking action. It is clear that the results, obtained both from road test data and through numerical simulations, are a combination of characteristics already observed in Figs 10 and

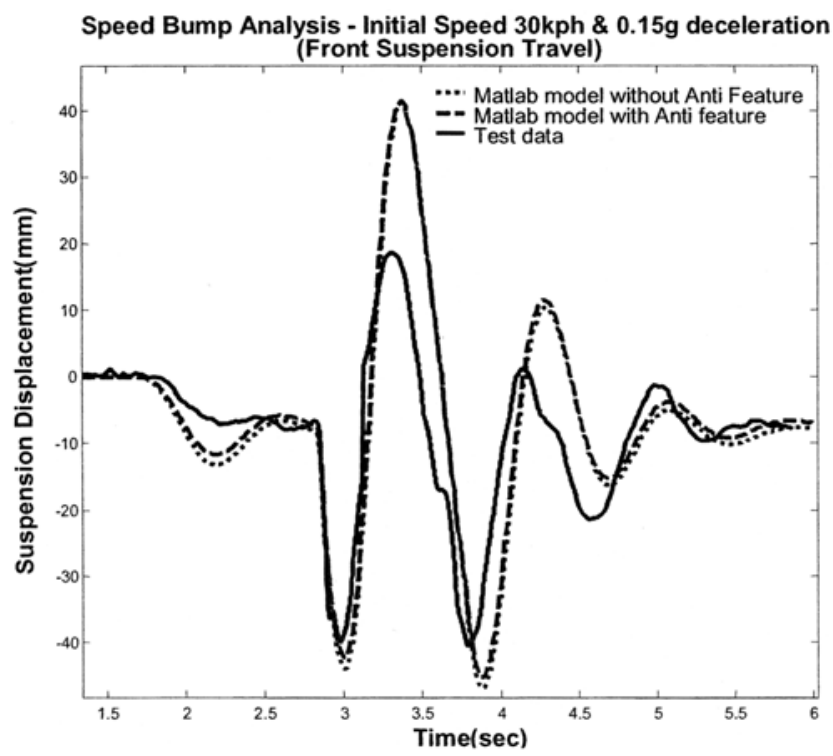

Fig. 14 Front suspension deflection 
12 or 13. The initial part of all the traces follows the characteristics in Fig. 10, again indicating that in a real-life situation the driver does not maintain a constant braking action (a natural reaction). Thus, the numerical results correspond to a slightly higher forward speed than the experimental value and, even given identical suspension characteristics, would have less of a dive posture. As a result, the suspension extension and deflection would be larger owing to higher inertial force transfer even with the same suspension characteristics. Furthermore, it is clear that the numerical results would be greater than the road test findings, although the total traverse time is very similar.

\section{CONCLUSION}

A number of conclusions can be made as a result of this study. Firstly, a relatively simple 6-DOF model (referred to as an intermediate model) can yield results of sufficient accuracy (typically within 20 per cent, given that the current intermediate model disregards the elastokinetics of the suspension system) that conform closely to road test data. The degree of conformity is clearly improved by the inclusion of other features, but sufficiently reliable predictions do not always require very sophisticated multi-body multidegree-of-freedom models

Secondly, pitch plane dynamics and pitch and bounce motions are non-trivial problems that are often incorrectly regarded as simple. Some driver behavioural characteristics inhibit perceived ideal conditions such as maintaining a constant braking action, which is often used in simulation studies. It is noteworthy that anti-dive and anti-squat features play a role in pitch plane dynamics, and their effect diminishes with any additional vehicle bounce, particularly at higher speeds. Increasing vehicle speed over a barrier caused, greater inertial imbalance, thus reducing the effect of anti-dive and anti-squat features which are designed essentially for normal pitch plane dynamics with smaller suspension vertical travel. This has been shown in the results of negotiating bumps at progressively higher forward speeds.

Finally, to replicate real-world conditions, attention should be paid to the elastokinetic behaviour of suspension systems which accounts for absorption of impact energies by distortion of structural members, thus reducing the observed differences between the ideal rigid-body simulation conditions and those experienced in practice.

\section{ACKNOWLEDGEMENTS}

The authors would like to express their gratitude to SIRIM BERHAD for the financial support it has extended to this research project, and to Ford Motor Company for technical and in-kind support. The effort of technical staff of the School of Aeronautical and Automotive Engineering, Loughborough University, is acknowledged.

\section{REFERENCES}

1 Dickinson, J. G. and Yardley, A. J. Development and application of a functional model to vehicle development IMechE Conference Transactions, 1993, Paper 930835.

2 Willumeit, H.-P., Neculau, M., Vikas, A. and Wohler, A. Mathematical models for the computation of vehicle dynamics behaviour during development. IMechE Conference Transactions, 1992, Paper 925046.

3 Sayers, M. W. and Han, D. S. A generic multibody vehicle model for simulating handling and braking. Veh. Syst. Dynamics, 1996, 25, 599-613.

4 Azman, M., Rahnejat, H. and Gordon, T. J. Suspension and road profile effects in vehicle pitch-plane response to transient braking and throttle actions. Dynamics of Vehicle on Roads and Track, 18th IAVSD, 2003.

5 Sharp, R. S. Influences of suspension kinematics on pitching dynamics longitudinal maneuvering. Veh. Syst. Dynamics, 1999, 33.

6 Rahnejat, H. Multi-Body Dynamics: Vehicle. Machines and Mechanisms, 1998 (Professional Engineering Publishing, Bury St Edmunds; Society of automotive Engineers, Warrendale, Pennsylvania).

7 Katz, A. Computational Rigid Vehicle Dynamics, 1997, (Krieger, Melbourne, Florida).

8 Gordon, T. J., Best, M. C. and Dixon, P. J. An automated driver based on convergent vector fields. Proc. Instn Mech. Engrs, Part D: J Automobile Engineering, 2002, 216(D4), 329-347. 